\title{
Manipulation of gene expression by an ecdysone-inducible gene switch in tumor xenografts Larry R Karns ${ }^{1}$, Anne Kisielewski²,4, Kathryn M Gulding3, Jabed M Seraj ${ }^{1}$ and Dan Theodorescu*3,5
}

Address: 1 Upstate Biotechnology Inc., Charlottesville, Virginia, USA, ${ }^{2}$ RheoGene Inc., Charlottesville, Virginia, USA, 3Department of Molecular Physiology and Biological Physics University of Virginia, Charlottesville, Virginia, USA, 4Upstate Biotechnology Inc., Charlottesville, Virginia, USA and 5Department of Molecular Physiology and Biological Physics University of Virginia, Charlottesville, Virginia, USA

E-mail: Larry R Karns - lkarns@upstate.com; Anne Kisielewski - lkisielewski@upstate.com; Kathryn M Gulding - Kgm3@virginia.edu; Jabed M Seraj - js7uw@virginia.edu; Dan Theodorescu* - theodorescu@virginia.edu

${ }^{*}$ Corresponding author

\begin{abstract}
Background: Rapid, robust and reversible induction of transgene expression would significantly facilitate cancer gene therapy as well as allow the in vivo functional study of newly discovered genes in tumor formation and progression. The popularity of the ecdysone inducible gene switch system has led us to investigate whether such a system can successfully regulate gene expression in a syngeneic tumor system in vivo.
\end{abstract}

Results: MBT-2 and Panc02 carcinoma cells were transfected with components of a modification of the ecdysone switch system driving firefly luciferase (F-Luc). In vitro luciferase expression \pm ecdysone analog GS-E indicated a robust induction with minimal baseline activity and complete decay after 24 hours without drug. In vitro selection of MBT-2 transfected cell clones which had complete absence of F-Luc expression in the absence of stimulation but which expressed this gene at high levels in response to GS-E were chosen for in vivo evaluation. Tumors from engineered MBT-2 cells were grown to $5 \mathrm{~mm}$ in diameter prior to GS-E administration, animals euthanized and tumors removed at 6, 12 and 24 hours after GS-E administration and assayed for F-Luc activity. GSE resulted in a maximal induction of F-Luc activity at 6 hours in tumor tissue with almost complete reversion to control levels by 12 hours.

Conclusions: This study is the first demonstration that robust and reversible transgene expression in tumors is feasible using the ecdysone system, allowing future rapid in vivo functional characterization of gene function or gene therapy applications.

\section{Background}

Rapid, robust and reversible induction of transgene expression would significantly facilitate certain applications of gene therapy and the study of cancer biology. Several inducible systems have been developed to regu- late gene expression at the transcriptional level in a temporal and quantitative manner. [1-4] These include systems based on heat shock, heavy metal ion, tetracycline, steroid, or ecdysone hormone induction. For in vivo applications, tetracycline, steroid, rapamycin and, 
ecdysone responsive systems have been studied most and in recent years. Currently the most popular inducible system is based on components of the tetracycline resistance operon, which has seen use in both tumors $[2,5]$ as well as transgenic animals. [6] However, these systems have limitations the most important being the significant background expression. Recently, the development of an inducible system based on the insect steroid ecdysone and the nuclear receptor that mediates its effect [7] has provided an alternative that may circumvent these limitations. The use of ecdysone inducible transgene expression in vitro $[8,9]$ and in transgenic mice [10] has recently been demonstrated. The use of this system in vivo has several potential advantages over other regulatory systems. The ecdysone hormones do not affect mammalian physiology like glucocorticoids or progestins and are not known to be toxic or teratogenic like tetracycline [11] allowing their use in both developmental studies with transgenic animals as well as in the context of gene therapy and tumor biology studies in mature animals.

The popularity of the ecdysone inducible gene switch system has led us to investigate its characteristics as a regulator of transgene expression in tumor xenografts since to our knowledge, this has not yet been reported. Prior to evaluating this in vivo, we have thoroughly examined the kinetics of this switch in vitro in 2 different tumor cell lines using 2 different reporters in order to evaluate the generality of these observations. The in vivo evaluation was carried out in a model of highly aggressive murine bladder cancer, which has previously been used for testing various therapeutic interventions. $[12,13]$ These bladder cell lines were transfected with a novel ecdysone system regulating the expression of firefly luciferase and then injected subcutaneously into syngeneic hosts. Treatment of the animals with ecdysone analog A/-(3-methoxy-2-ethylbenzoyl)-A/'-(3,5dimethylbenzoyl)-A/'-tert-butyl hydrazine (GS-E) was then carried out with subsequent evaluation of in vivo gene expression. Our data suggests that in vivo induction of tumor transgene expression is feasible and reversible using an ecdysone switch and opens the avenue for the efficient functional characterization of many newly discovered genes as well as novel gene therapy approaches.

\section{Results \\ Derivation of mouse tumor cell lines containing a novel gene regulatory mechanism}

A description of the plasmids encoding the two components of an ecdysone inducible transcription activation system was reported previously [14]. The plasmids pGAL4-EcR and pVP16-mRXR encode proteins which heterodimerize and regulate transcription upon the addition of ecdysone type ligands (Figure 1A). Using these plasmids as a starting point, a novel indicator of the inducible gene expression system was constructed as follows. The CMV promoter and enhancer were removed from the mammalian expression vector pCI-neo (Promega). Inserted in its place were five copies of the GAL4 binding site followed by the minimal promoter region of the major late promoter from adenovirus (from pG5-luc (Promega)) and the coding region for the enhanced green fluorescent protein (EGFP) gene (from pEGFPN1) (Clontech). The control plasmid, pRL-TK (Promega), expresses the Renilla luciferase gene under the control of the HSV thymidine kinase (TK) promoter. This plasmid provides constitutively high gene expression and we use it as an indicator of the number of tumor cells present in the tissue samples harvested from mice in the in vivo experiments.

MBT-2 cells, a mouse urothelial cancer cell line, or Panco2, a mouse ductal adenocarcinoma, were transfected by electroporation with a mixture of the four plasmids; the two components of the gene expression system, pVP16-mRXR and pGAL4-EcR; the indicator and selection plasmid, pGAL4-EGFP-SV4O-neo; and the internal control plasmid, pRL-TK. Stably transfected MBT-2 cells were selected in media containing $500 \mu \mathrm{g} /$ $\mathrm{ml} \mathrm{G418}$ and Panco2 cells in $700 \mu \mathrm{g} / \mathrm{ml} \mathrm{G} 418$ for three weeks after which there were many large cell colonies on each transfection plate. The drug resistant cells were harvested by trypsinization, pooled, and cloned to single cells by limiting dilution in 96 well plates. When colonies had grown in a subset of the wells in the 96 well plate, fresh media containing GS-E was added to all wells to a final GS-E concentration of $5 \mu \mathrm{M}$. Cells were treated for 24 hours and all wells were visualized with the fluorescent microscope to detect EGFP expression.

At least 96 clones of MBT-2 or Panco2 clones demonstrating fluorescence were trypsinized and expanded into 24 well plates. When the clones had reached at least $50 \%$ confluence in the 24 well plate, GS-E was again added to $5 \mu \mathrm{M}$ to verify the earlier observation of induced EGFP expression. Four clones of each cell line exhibiting high levels of EGFP expression were expanded for further analysis. Each cell line was transiently transfected with $1 \mu \mathrm{g}$ pFR-luc and carrier DNA. Luciferase activity in the DMSO treated wells was similarly low in all wells of the triplicates of the eight transfections. After treatment with $15 \mu \mathrm{M}$ GS-E, three of the four MBT-2 clonal cell lines had a two-fold or lower increase in luciferase activity. Clone \#2 had 8.4-fold higher luciferase activity with GS-E treatment. This clone, designated MBT2+RheoSwitch-2, was used for further development. In a similar analysis, Panco2+RheoSwitch-3 and -4 demonstrated induction levels of 366- and 428-fold respectively. No 


\section{$\mathbf{A}$ pVP16-mRXR}

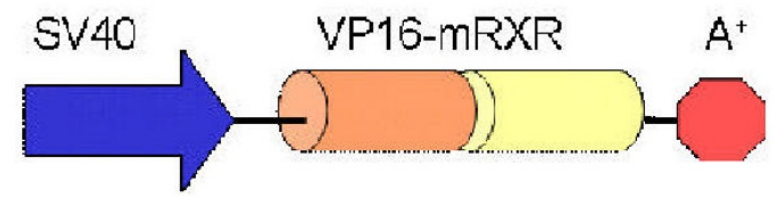

pGAL4-EcR

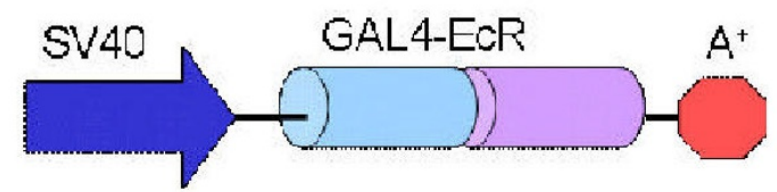

GAL4-EGFP +SV40-neo

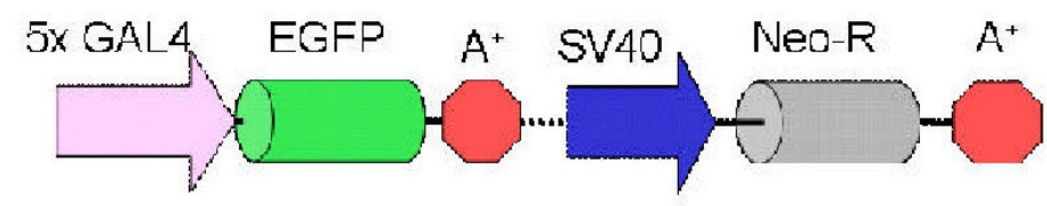

pRL-TK

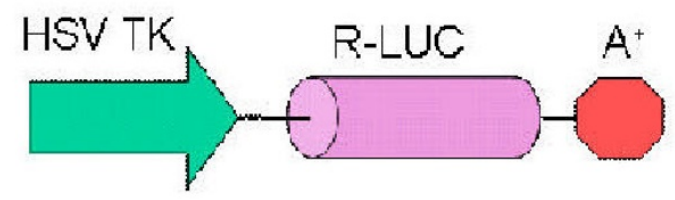

\section{B \\ pG5-Iuc}

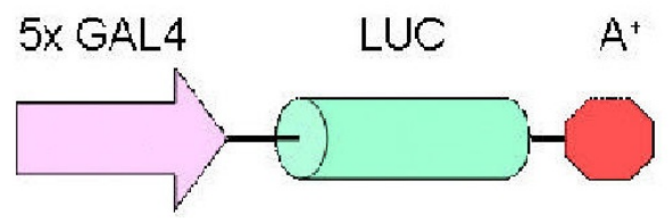

pGAL4-SEAP

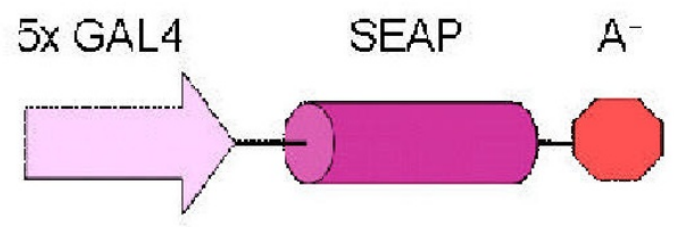

\section{pSV40-Zeo}

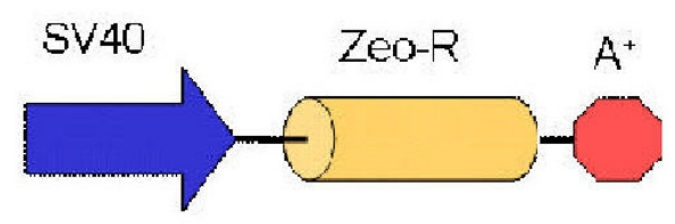

Figure I

Plasmid constructs used in cell line generation.(A) Plasmids used in generating RHeoSwitch Cell Lines.(B) Reporter Plasmids Introduced into RHeoSwitch Cells 
significant change in the Renilla luciferase activity was observed with GS-E treatment in all cell lines.

\section{Stable introduction of inducible reporter genes into the RHeoSwitch tumor cell lines}

The MBT-2+RHeoSwitch-2 cell line containing the RHeoSwitch expression system was used for the introduction of two different reporter gene systems. The plasmid, pG5-luc, contains the GAL4 response element expressing the luc+ gene (Figure 1B). The vector pGAL4-SEAP containing the GAL4 response element and the coding region for the SEAP protein, a unique, second reporter gene, was also used. Each of these vectors was linearized and co-transfected with linearized pSV40-Zeo by electroporation into the MBT-2+RHeoSwitch-2 cells. Cells were selected in media containing $400 \mu \mathrm{g} / \mathrm{ml}$ Zeocin until colonies appeared in each of the plates. Zeocin resistant cells were trypsinized, pooled, and single cell cloned by limited dilution in 96 well plates. Aliquots of the pooled cells used for cloning were also plated in triplicate, treated with GS-E or DMSO for $24 \mathrm{hrs}$. Assays for the appropriate reporter in each cell pool showed induction ratios of 17-fold for the luciferase transfected cells and 22-fold for the SEAP transfected cells. This verified the presence of the GS-E inducible reporter genes (luciferase or SEAP) in the cloned pool of cells.

Clones were grown in Zeocin containing media until colonies appeared. Eighty-four luciferase transfected clones and 113 SEAP transfected clones were transferred from the 96 well plates and duplicate wells of each were treated with either DMSO or $15 \mu \mathrm{M}$ GS-E in DMSO for 24 hours. Each pair of wells was assayed for reporter protein activity and clones demonstrating basal levels of activity in the DMSO treated sample and high levels in the GS-E sample were further expanded for additional testing. These cell clones were plated in a six well plate and triplicate wells were treated with DMSO or $15 \mu \mathrm{M}$ GS-E for 48 hours. Luciferase clone MBT-2+RHeoSwitch-2+luc$5 \mathrm{~B} 3$ was chosen to be used for a more detailed analysis of the properties of the gene induction system.

The inducible reporter gene plasmids, pG5-luc and pGAL4-SEAP, were transfected into the Panco2+RHeoSwitch- 3 and -4 cells and stable transfectants were isolated as they were into the MBT-2+RHeoSwitch cells. Multiple Zeocin resistant cell clones from the four transfections (36 Panco2+RHeoSwitch-3+luc, 75 Panco2+RHeoSwitch-4+luc, 8 Panc02+RHeoSwitch3+GAL4-SEAP, 98 Panco2+RHeoSwitch-4+SEAP) were plated in duplicate and treated with DMSO or $15 \mu \mathrm{M}$ GSE. A sample of clones with basal level of reporter in the DMSO treated sample and the highest level of reporter in the GS-E treated sample were selected to be expanded and further tested.
Multiple assays in triplicate on the GAL4-SEAP transfected clones gave induction levels ranging from 118- to 551-fold for the best five Panco2+RHeoSwitch-3 clones and from 52- to 486-fold for the best twelve Panc02+RHeoSwitch-4 clones. Clones Panco2+RHeoSwitch-3+GAL4-SEAP-5 and -8 and Panco2+RHeoSwitch-4+GAL4-SEAP-8 and -9 were used for further characterization. Multiple assays in triplicate on the pG5-luc transfected clones yielded induction levels ranging from 32- to 51-fold for the four best Panco $2+$ RHeoSwitch-3 clones and from 7- to 94-fold for the four best Panco2+RHeoSwitch-4 clones. Clones Panco2+RHeoSwitch-3+luc-3Cl AND -1B1 and Panco2+RHeoSwitch4+luc-3A1 and -4B3 were used for further characterization. These clones do not exhibit the high levels of induced GFP expression that was seen in the initial RHeoSwitch transfected cells. This may be explained by the fact that they were selected on the basis of the induction level of the reporter gene expression, however, contrary to the initial screen, the level of induction of GFP was not used as a selection criteria and cell clones in which the expression of GFP has variegated were isolated. A summary flowchart of the cell line selection and characterization is shown in figure 2.

\section{Characterization of the novel gene regulatory mechanism in vitro}

Treatment of luciferase clone $5 \mathrm{~B} 3$ with $15 \mu \mathrm{M}$ GS-E for 48 hours consistently induced luciferase levels from near background with only DMSO added, to nearly 150-fold (Table 1) when adjusted for non specific R-Luc induction which was approximately $\mathbf{1 . 2}$ fold. In order to understand the induction kinetics of the inducible system, cell clone MBT-2+RHeoSwitch-2+luc-5B3 was treated with GS-E and cells were harvested at different times to determine the time course of gene induction. At each time point, the control and treated cells were collected in passive lysis buffer and dual luciferase assays performed. Data were plotted as a GS-E (RLU1/RLU2)/Control (RLU1/RLU2) ratio (as in Table 1) fold induction over the $\mathrm{o}$ time point which was set empirically as 1 . This data shown in Figure 3A indicates that gene expression is increased by 8 hours and reaches maximum induction by 24 hours. The internal control, Renilla luciferase, increase steadily with time, reflecting the increase in cell number, but the increase was the same with DMSO or GS-E treatment (raw data not shown but effect accounted for in the calculation as defined above).

The sensitivity of the gene expression system to varying doses of GS-E was determined by treating duplicate samples of clone MBT-2+RHeoSwitch-2+luc-5B3 with varying concentrations of GS-E for 24 or 48 hours and dual luciferase assays were performed on the cell lysates (Figure $3 \mathrm{~B})$. Data were plotted as a GS-E(RLU1/RLU2)/Con- 


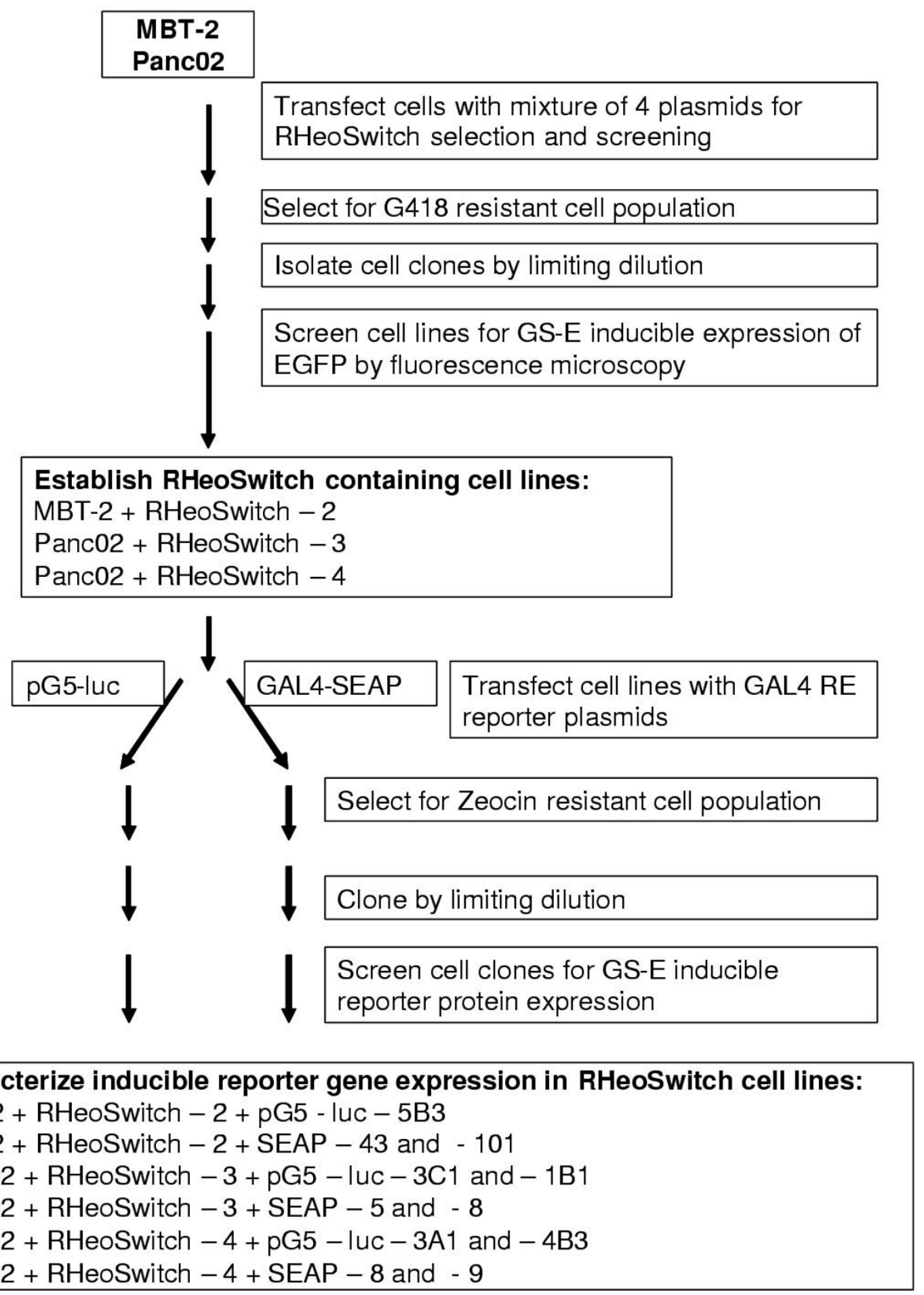

Figure 2

A summary flowchart of the cell line selection and characterization 

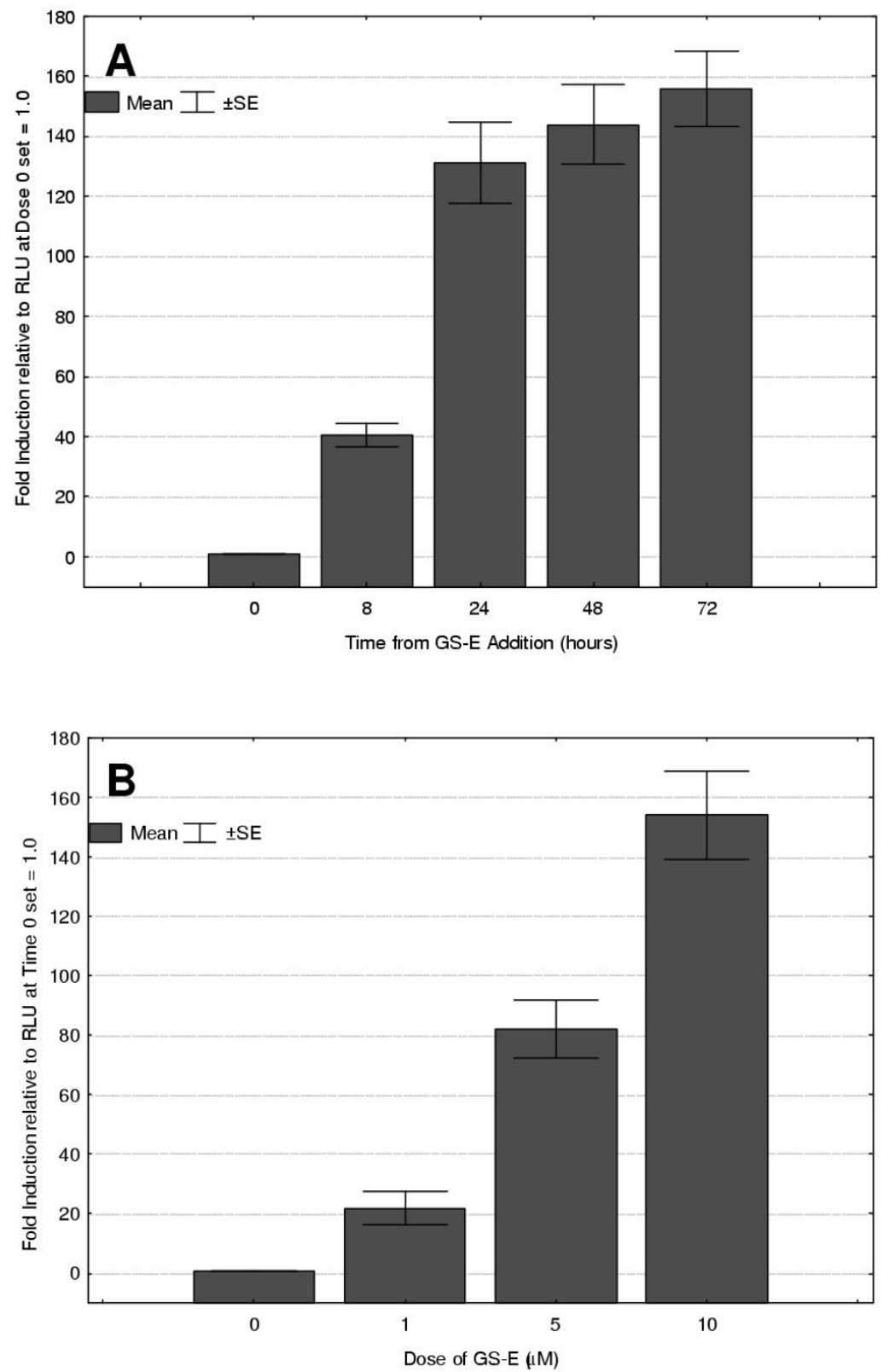

Figure 3

Time and dose response kinetics of luciferase in response to GS-E.(A) Cell clone MBT-2+RHeoSwitch-2+luc-5B3 was treated with GS-E and cells were harvested at different times to determine the time course of gene induction. At each time point, the control and treated cells were collected in passive lysis buffer and dual luciferase assays performed. Data were plotted as a GS-E (RLUI/RLU2)/ Control (RLUI/RLU2) ratio (as in Table I) fold induction over the 0 time point which was set empirically as I. The internal control, Renilla luciferase, increase steadily with time, reflecting the increase in cell number, but the increase was the same with DMSO or GS-E treatment (raw data not shown but effect accounted for in the calculation as defined above).(B) The sensitivity of the gene expression system to varying doses of GS-E was determined by treating duplicate samples of clone MBT-2+RHeoSwitch-2+luc-5B3 with varying concentrations of GS-E for 24 hours and dual luciferase assays and data analysis were performed on the cell lysates as described in $(A)$ above. 
Table I: Summary of GS-E induction of luciferase activity in clone MBT-2 + RHeoSwitch \#2 + pGS-luc - 5B3

\begin{tabular}{|c|c|c|c|c|c|c|c|c|c|}
\hline \multirow[t]{2}{*}{ Experiment } & \multirow{2}{*}{$\begin{array}{l}\text { RLUIA } \\
\text { CON }\end{array}$} & \multicolumn{2}{|l|}{ RLUI } & \multirow{2}{*}{$\begin{array}{l}\text { RLU2' } \\
\text { CON }\end{array}$} & \multicolumn{2}{|l|}{ RLU2 } & \multirow{2}{*}{$\begin{array}{l}\text { RLUI/RLU2 } \\
\text { CON }\end{array}$} & \multicolumn{2}{|l|}{ RLUI/RLU2 } \\
\hline & & GS-E & T/C & & GS-E & T/C & & GS-E & $\mathbf{T} / \mathbf{C}$ \\
\hline$A$ & $4457^{*}$ & 992343 & 222.6 & 543227 & 678517 & 1.2 & 0.01 & 1.46 & 178.3 \\
\hline B & 4123 & 948272 & 230.0 & 579818 & 709519 & 1.2 & 0.01 & 1.34 & 188.0 \\
\hline C & 4771 & 982058 & 205.8 & 658164 & 823060 & 1.3 & 0.01 & 1.19 & 164.6 \\
\hline $\mathrm{D}$ & 3915 & 597044 & 152.5 & 537789 & 720480 & 1.3 & 0.01 & 0.83 & 113.8 \\
\hline $\mathrm{E}$ & 1757 & 350947 & 199.7 & $756 \mid 4$ & 95031 & 1.3 & 0.02 & 3.69 & 158.9 \\
\hline $\mathrm{F}$ & 1405 & 314349 & 223.7 & 232147 & 300061 & 1.3 & 0.01 & 1.05 & 173.1 \\
\hline G & 1343 & 234858 & 174.9 & | 74883 & 207357 & 1.2 & 0.01 & 1.13 & 147.5 \\
\hline $\mathrm{H}$ & 3958 & 737902 & 186.4 & 197599 & 227480 & 1.2 & 0.02 & 3.24 & 161.9 \\
\hline 1 & 3822 & 710260 & 185.8 & 209591 & 251976 & 1.2 & 0.02 & 2.82 & 154.6 \\
\hline \multirow[t]{4}{*}{ J } & 3714 & 692231 & 186.4 & 218191 & 259666 & 1.2 & 0.02 & 2.67 & 156.6 \\
\hline & & Mean & 196.8 & & Mean & 1.2 & & Mean & 159.7 \\
\hline & & SD & 24.4 & & SD & 0.1 & & SD & 20.1 \\
\hline & & $\mathrm{N}$ & 10 & & $\mathrm{~N}$ & 10 & & $\mathbf{N}$ & 10 \\
\hline
\end{tabular}

CON: control carrier applied only Only 48 hour time-points displayed in this summary ${ }^{\wedge}$ RLUI is expression of firefly luciferase from the inducible promoter 'RLU2 is expression of Renilla luciferase from the constitutive TK promoter *All data points shown are the average from duplicate or triplicate samples

trol(RLU1/RLU2) ratio (as in Table 1) fold induction over the o drug concentration which was set empirically as 1. These results show an increase in gene inducing activity with increasing GS-E concentrations up to $10 \mu \mathrm{M}$. A significant increase was seen with as little as $1 \mu \mathrm{M}$ GSE. No change was seen in the level of the internal control Renilla luciferase with varying concentrations of GS-E (raw data not shown but effect accounted for in the calculation as defined above).

The decay characteristics of the induction were also studied. After a 24 hour incubation, the medium containing GS-E was removed and the cells washed three times with complete medium without GS-E. The cells were then re incubated for various durations. At set intervals replicate wells were removed and dual luciferase assays were performed on the cell lysates (Figure 4A). This data indicates that the GS-E mediated induction is completely reversible and that this occurs within 48 hours.

Similar dose response and time course of induction of gene expression experiments were performed on the Panco2 cells containing the RHeoSwitch and the inducible reporter genes. The time course of gene induction demonstrated for four independent Panco2+RHeoSwitch+luciferase cell clones each showed an increase within 12 hours of GS-E treatment and reached the maximum induction by 24 hours. Four independent Panco2+RHeoSwitch + SEAP clones also induced expression by 12 hours but the level of SEAP activity continued to increase for 72 hours, possibly due to the accumulation of the enzyme in the cell culture media over the entire treatment time. Treatment of five of these independent Panco2+RHeoSwitch+reporter gene clones with varying doses of GS-E consistently yielded a significant induction in gene expression with $1 \mu \mathrm{M}$ GS-E (23.5-fold induction over DMSO treatment alone) and a maximal induction with $15 \mu \mathrm{M}$ GS-E (80.2-fold induction over DMSO). The dose response and time course of induction in the Panco2 cells were similar to the results seen in experiments on the MBT-2 cells. These results show that the RHeoSwitch gene induction system gives consistent results independent of the cell type being used, the reporter gene used to assess the activity, as well as the chromosomal integration site of the DNA encoding the RHeoSwitch components (compare Panco2+RHeoSwitch-3 and -4 described above) or the integration site of the reporter gene DNA (compare multiple reporter clones from each of three RHeoSwitch clones described above).

\section{In vivo results}

The potency and favorable kinetics of GS-E as an ecdysone-system inducer in vitro in both the pancreatic and bladder cell lines led us to evaluate its performance in vivo in a bladder xenograft model. To measure the pharmacokinetics of GSE mediated gene induction in animals, we inoculated the MBT-2 murine bladder cancer cell line into syngeneic mice and after the tumors reached $0.4-0.5 \mathrm{~mm}$ in diameter, the mice were treated 

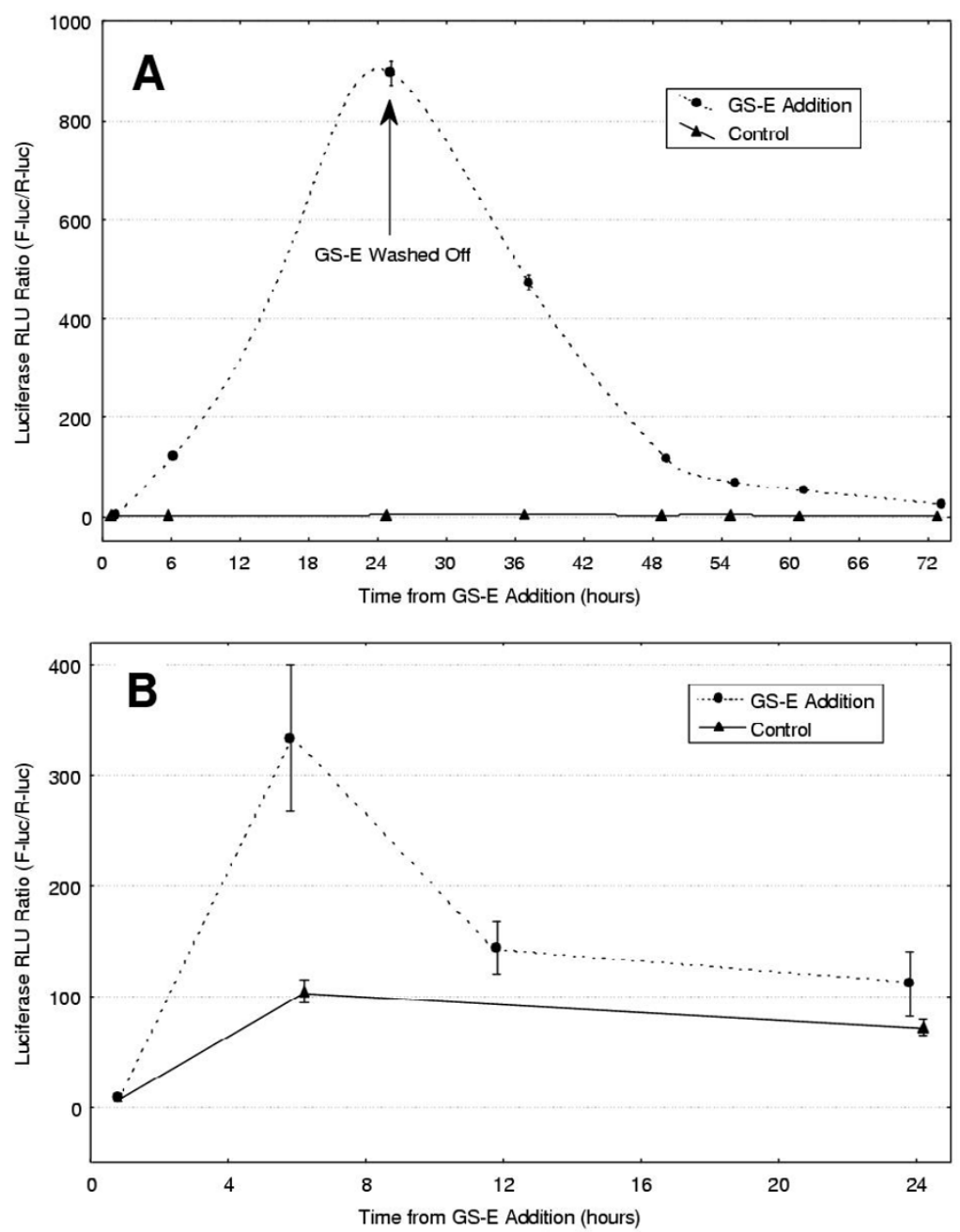

\section{Figure 4}

(A) The decay characteristics following initial induction of luciferase by GS-E. After a 24 hour incubation using similar conditions to those described in figure A, the medium containing GS-E was removed and the cells washed three times with complete medium without GS-E. The cells were then re incubated for various durations as shown in the graph. At set intervals triplicate wells were removed and dual luciferase assays were performed on the cell lysates. Error bars indicate mean $+/-$ one standard error for triplicate experiments. (B) In vivo induction of gene expression in tumor xenografts. Six-to-8 weekold $\mathrm{C} 3 \mathrm{H} / \mathrm{He}$ origin mice were given injections in the anterior flank ( 2 sites per mouse) with $10^{6}$ MBT-2 tumor cells suspended in $0.1 \mathrm{ml}$ of serum free medium. Mice were examined every week, and tumors were measured with calipers in the greatest 2 diameters. When tumors reached $5 \mathrm{~mm}$ in greatest diameter, mice were injected intraperitoneally with either 45 milligrams of GS-E dissolved in $90 \mu \mathrm{l}$ of DMSO and $360 \mu \mathrm{l}$ of sesame oil (GS-E Addition) or with equivalent volumes of DMSO and $360 \mu$ l of sesame oil alone (Control). Cohorts of mice were euthanized at various time points following drug administration or vehicle administration and tumors processed for luciferase activity as described in the materials and methods section. Error bars indicate mean $+/$ - one standard error for 4-6 tumors. 
with GS-E. At 6,12 and 24 hour intervals following inoculation, the tumors were excised and analyzed for luciferase induction. Luciferase protein was chosen primarily because its 3-4 hour half-life in mammalian cell allows precise kinetic studies [15]. The renilla luciferase was used as an internal control in addition to untreated mice. Data shown in Figure $4 \mathrm{~B}$ indicates that induction of luciferase activity in tumor xenografts is prompt, peaking between 6-12 hours. In addition, decay of the effect occurs swiftly and baseline expression levels are reached by $12-24$ hours. Although available to us, the identification of cell specific expression within the tumor using the induction of GFP in these tumor cells was complicated by the decrease in the level of GFP expression (data not shown), presumably due to position effect variegation of the transfected DNA as well as poor quantitation ability.

\section{Discussion}

Methods devised to control gene expression must allow for rapid, robust, precise, and reversible induction of gene activity. Because steroids are small lipophilic molecules that penetrate all tissues and are quickly metabolized and cleared, they appear to be ideal inducers for developing gene regulation systems that fulfill these criteria. To exploit the pharmacokinetics of steroids while eluding the potential complications of the use of a mammalian hormone as the inducer, the Evans laboratory developed an inducible system based on the insect steroid ecdysone and the nuclear receptor that mediates its effects. [7] This system is comprised of a chimeric protein composed of the VP16 activation domain $(\mathrm{Vg})$ fused to an ecdysone receptor (EcR) with altered DNA-binding specificity heterodimerizes with the retinoid $\mathrm{X}$ receptor (RXR) and binds a unique synthetic response element not recognized by natural nuclear hormone receptors. Upon exposure to the ecdysone analog muristerone A (murA), the VgEcR/RXR complex efficiently induces the expression of reporter genes in mammalian cells and transgenic animals. In the RHeoSwitch gene expression system, the properties of the chimeric receptors have been optimized through the analysis of a series of truncation and site-directed mutations (Palli, et.al., manuscript in preparation). This study demonstrates the utility of a first generation system in vitro and in vivo and subsequent modified versions of the RHeoSwitch will be sensitive to lower concentrations of ligand and result in higher levels of induced gene expression (see [www.RHeoGene.com] ).

The popularity of the ecdysone-inducible gene switch led to the unexpected rapid depletion of murA stocks, the primary inducer used in the system. MurA was isolated in the early 1970s from the seeds of kaladana (Ipomoea calonyction), a plant native to the southern slopes of the
Himalayas. [10] The difficulty in obtaining kaladana seeds prompted efforts to seek alternative inducers derived from more common plants or synthetic analogs such as GS-E. GS-E is one of a family of diacylhydrazines that have been found to act as non-steroidal ecdysone mimics that can function as gene inducers [16].

The avalanche of new genes resulting from completion of the genome project has accentuated the need to develop methods to determine gene function. The requirement for tight regulation, easy titration and minimal background expression is particularly important in the study of genes such as tumor suppressors or cell cycle regulatory genes for which cell lines stably expressing the product can rarely be made. In addition, deliberate manipulation of gene activity is also an indispensable tool for clinical applications such as gene therapy. The strengths of the ecdysone-inducible system appear to be its tight regulation, its dose responsiveness, and the favorable uptake and clearance kinetics of the inducer, which results in rapid gene switching. Thus it appears ideal for both the in vitro and in vivo study of newly characterized genes in their role in tumor formation and progression. In vitro data indicates that our constructs when used in combination with GS-E, offer an excellent induction profile and rapid decay of gene expression when the compound is removed from in vitro culture. Importantly, we have demonstrated similar kinetics for 2 different cancer cell lines with 2 different reporters (F-luc and SEAP) thus validating the generality of the observed results. In addition, these constructs are also associated with a low baseline gene expression level allowing the study of genes whose expression may be incompatible with the generation of constitutively expressing clones. A recent report showed that the expression of nonmuscle myosin heavy chain-B induced by Muristerone A in vitro [8] suppressed the subsequent tumorigenicity of the implanted cells in vivo.

The potency and favorable kinetics of the cell lines we developed in conjunction with GS-E as an ecdysone-system inducer in vitro in both the pancreatic and bladder cell lines led us to evaluate the performance of this system in vivo in a bladder cancer xenograft model. The advantages of the MBT-2 murine bladder tumor model are several fold. First, it was derived by chemical carcinogenesis, the most common known etiologic factor in bladder cancer. Second, when inoculated orthotopically it reproduces the biology of human muscle invasive bladder cancer. Thirdly, it has been widely used as a model system of various therapeutic interventions in bladder cancer. $[12,13]$ Interestingly, our data indicates that the kinetics of induction of tumor luciferase in response to GS-E mirror those observed with transgenic mice in response to Po$\mathrm{nA}$. [10] This finding may indicate that despite their use 
in a malignant context, the ecdysone constructs behave with similar kinetics to that in normal cells thus implying a general utility in studies of various histological tumor types. In addition, comparison of the in vivo (Figure 4B) and in vitro (Figure $3 \mathrm{~A}$ ) induction curves reveals roughly similar time to peak induction despite the fact that the in vivo experiments were carried out with a mixture of GSE in sesame seed oil, which purportedly slows its delivery. [9]

Although available to us, the identification of cell specific expression within the tumor using the induction of GFP in these tumor cells was complicated by the decrease in the level of GFP expression, presumably due to position effect variegation of the transfected DNA. In addition, the utility of GFP for the localization of gene expression in vivo, particularly in conditions of hypoxia, such as tumors, has been recently been questioned [17]. Finally, quantitative analysis of tumor GFP induction is difficult and less reliable than other reporters such as luciferase. Therefore we have limited our in vivo experiments determining the general level of gene induction within the tumor by using a luciferase reporter which has been proven to be reliable in vivo.

\section{Conclusions}

We have used a modified ecdysone reporter system which has favorable baseline and induction kinetics in response to a novel ecdysone analog GS-E in murine tumors in vitro. We have demonstrated the general applicability of this system by demonstrating its use in two different mouse tumor cell lines, integration of the RHeoSwitch DNA components at multiple chromosomal locations, as well as with 2 different gene reporters, luciferase, and SEAP. We have presented data that is the first demonstration of tumor cell lines stably transfected with an ecdysone inducible constructs tested in vivo demonstrating that in vivo these constructs coupled with GS-E allow rapid and robust induction of gene expression in tumor xenografts. Finally, due to the rapid decay of gene expression following maximal activation, this system can be easily titrated for the duration of the induction necessary by using slow release formulations such as ALZET mini pumps [18]. The postgenomic era has accentuated the need to develop methods to determine in vivo gene function in cancer and this ecdysone system may be a useful approach to address this.

\section{Materials and Methods}

\section{Cell culture and plasmid DNA constructs}

MBT-2 murine urothelial cancer cells and their culture conditions for passage were previously described. [12] The murine ductal cell pancreatic adenocarcinoma cell line, Panco2 [19], was kindly provided by Dr. James Nelson, MD Anderson Cancer Center. Plasmid DNA was prepared by the "EndoFree Plasmid Maxi kit" from Qiagen (Valencia, CA) using the manufacturer's procedure. Cells were simultaneously co-transfected by electroporation (Gene Pulser, Bio-Rad Inc.) with pGAL4-EcR, pVP16-mRXR, pRL-TK (Renilla luciferase) and pGAL4EGFP. The plasmids pGAL4-EcR encodes a fusion protein between the yeast GAL4 DNA binding domain and the ligand binding domain of the ecdysone receptor from the spruce budworm, Choristoneura occidentalis. Plasmid pVP16-mRXR encodes a fusion protein between the transcriptional transactivator VP16 from Herpes simplex virus (HSV) and the mouse RXR protein. The vector pRL-TK (Promega), expressing the Renilla luciferase enzyme from the HSV thymidine kinase (TK) promoter, was included as an uninduced constitutively expressed internal control. The vector pGAL4-EGFP was constructed from pCI-neo (Promega) by replacing the CMV promoter and enhancer with a fragment from $\mathrm{pG}_{5}$-luc (Promega) containing the $5 \times$ GAL4 recognition sequences and the minimal promoter from the adenoviral late promoter and the EGFP gene from pEGFP (Clontech). In addition to the GAL4-EGFP expression cassette, this plasmid contains the neomycin resistance gene under the control of the SV4O promoter and enhancer for selection of transfected cells in G418.

\section{Transfection and isolation of clones stably transfected with inducible reporter gene plasmids}

Cells were transfected by electroporation with mixtures of the four plasmids which had been linearized by restriction enzyme digestion. Following transfection, cells were allowed to recover for $24 \mathrm{~h}$ prior and were then selected in $500 \mu \mathrm{g} / \mathrm{ml} \mathrm{G} 418$ for MBT-2 cells and $700 \mu \mathrm{g} / \mathrm{ml}$ G418 for Panco2 cells for three weeks. Cells were then cloned by limited dilution into 96 well plates. Expression of green fluorescent protein (GFP) was induced by addition of GS-E (A/-(3-methoxy-2-ethylbenzoyl)-A/'-(3,5dimethylbenzoyl)-A/'-tert-butylhydrazine). GS-E is a member of the bisacylhydrazine chemical family which has been shown to have no adverse effects over a broad dosage range in mammals. [11] The 96 well plates were visualized under a fluorescent microscope before and after addition of the compound. Clones exhibiting low background of green fluorescence prior to addition of the compound and high levels following the addition were selected for further transfections.

Cell clones were transiently transfected using Superfect reagent with $1 \mu \mathrm{g}$ per well of 6 well plate with either $\mathrm{pFR}$ luc (Promega) or pGAL4-SEAP (secreted alkaline phosphatase) (provided by Dr. R. Herzig, Rheogene Inc.) and $3 \mu \mathrm{g}$ carrier DNA (pLITMUS, New England Biolabs). To three wells, DMSO was added to a final concentration of $0.3 \% \mathrm{v} / \mathrm{v}$. GS-E in DMSO was added to the remaining three wells to a final GS-E concentration of $15 \mu \mathrm{M}$ and 
DMSO concentration of $0.3 \%$. Cells were treated for 24 hours, at which time they were collected in Passive Lysis Buffer and the activity of firefly and Renilla luciferase in the cell extract was determined. The clone exhibiting the highest level of induced gene expression was then used for further development. These cells where in turn cotransfected with pSV4o/Zeo2 (Invitrogen) and pG5-luc (Promega), encoding firefly luciferase or pGAL4-SEAP and MBT-2 cells were selected in $400 \mu \mathrm{g} / \mathrm{ml}$ Zeocin and Panco2 cells in $500 \mu \mathrm{g} / \mathrm{ml}$ Zeocin. Zeocin resistant cells were then cloned by limiting dilution into 96 well plates. Replica plates were then created and induction of luciferase activity in response to GS-E carried out as for GFP. Clones exhibiting low background luciferase expression prior to addition of the compound and high levels following the addition were selected for further in vivo and in vitro study.

\section{Measurement of reporter protein levels in vitro and in vivo}

To determine the luciferase activity, cells or tissues were lysed in Passive Lysis Buffer (Promega) and assayed for firefly (F-luc) and Renilla (R-Luc) luciferase according to the Promega Luciferase assay kit instructions in a Turner Designs (Sunnyvale, CA) TD-20/20 or a PharMingen Monolight 3010 luminometer. The luciferase activity was recorded as Relative Light Units (RLU).

For the determination of SEAP levels, cells were treated with DMSO or GS-E for 48 hours. Media was removed from all cultures and either assayed or stored at -20 degrees until assayed. Assays were performed using the chemiluminescent SEAP assay procedure as described by the manufacturer in the Great EscAPe SEAP Assay system (Clontech). The chemiluminescent signal was measured with a PharMingen Monolight 3010 luminometer and recorded as Relative Light Units (RLU).

\section{In vivo tumor assays}

Six-to-8 week-old $\mathrm{C}_{3} \mathrm{H} / \mathrm{He}$ origin mice were obtained from Taconic Farms [www.taconic.com] and maintained strictly according to approved Institutional Animal Care and Use Committee protocols. Food and water were provided ad libitum. Cohorts of mice were given injections in the anterior flank ( 2 sites per mouse) with $10^{6}$ tumor cells suspended in $0.1 \mathrm{ml}$ of serum free medium. Mice were examined every week, and tumors were measured with calipers in the greatest 2 diameters. When tumors reached $5 \mathrm{~mm}$ in greatest diameter, mice were injected intraperitoneally with either 45 milligrams of GS-E dissolved in $90 \mu \mathrm{l}$ of DMSO and $360 \mu \mathrm{l}$ of sesame oil or with equivalent volumes of DMSO and $360 \mu \mathrm{l}$ of sesame oil alone. Cohorts of 3 mice were euthanized 6,12 and 24 hours following drug administration and cohorts of 2 mice euthanized 6 and 24 hours following vehicle administration and tumors processed for luciferase activity as described above. To evaluate the reporter induction, the ratio of RLU from firefly (F-luc) and Renilla (R-Luc) luciferase was evaluated (RLU\{F-luc\}/RLU\{R-luc\}). This time course was based on the pharmacokinetics of PonA following this route of administration. [10]

\section{Statistical analysis}

All in vitro assays were performed using duplicate or triplicate samples and error bars represent one standard error from the mean. Figures consist of a representative experiment carried out at least $2-3$ times with similar results. Data were plotted and statistical analysis was accomplished using the STATISTICA for Windows computer program (StatSoft, Inc. Tulsa, OK).

\section{Acknowledgments}

This work was supported in part by NIH grant CA075 I I5-04 and Kimmel Foundation awards to D.T.

\section{References}

I. Gingrich JR, Roder J: Inducible gene expression in the nervous system of transgenic mice. Annu Rev Neurosci 1998, 2 I:377-405

2. Gossen M, Freundlieb S, Bender G, Muller G, Hillen W, Bujard H: Transcriptional activation by tetracyclines in mammalian cells. Science 1995, 268: I766-1769

3. Saez E, No D, West A, Evans RM: Inducible gene expression in mammalian cells and transgenic mice. Curr Opin Biotechnol 1997, 8:608-6I6

4. Harvey DM, Caskey CT: Inducible control of gene expression: prospects for gene therapy. Curr Opin Chem Biol 1998, 2:5I 2-5 I8

5. Senner V, Sturm A, Hoess N, Wassmann H, Paulus $W$ : In vivo glioma model enabling regulated gene expression. Acta Neuropathol (Berl) 2000, 99:603-608

6. Furth PA, St Onge L, Boger H, Gruss P, Gossen M, Kistner A, Bujard $H$, Hennighausen L: Temporal control of gene expression in transgenic mice by a tetracycline-responsive promoter. Proc Natl Acad Sci USA 1994, $91: 9302-9306$

7. No D, Yao TP, Evans RM: Ecdysone-inducible gene expression in mammalian cells and transgenic mice. Proc Natl Acad Sci USA 1996, 93:3346-335।

8. Yam JW, Chan KW, Hsiao WL: Suppression of the tumorigenicity of mutant p53-transformed rat embryo fibroblasts through expression of a newly cloned rat nonmuscle myosin heavy chain-B. Oncogene 200I, 20:58-68

9. Hoppe UC, Marban E, Johns DC: Adenovirus-mediated inducible gene expression in vivo by a hybrid ecdysone receptor. $\mathrm{Mol}$ Ther 2000, I:159-164

10. Saez E, Nelson MC, Eshelman B, Banayo E, Koder A, Cho GJ, Evans RM: Identification of ligands and coligands for the ecdysoneregulated gene switch. Proc Natl Acad Sci USA 2000, 97:I45I214517

II. Dhadialla TS, Carlson GR, Le DP: New insecticides with ecdysteroidal and juvenile hormone activity. Annu Rev Entomol 1998 43:545-569

12. Eto M, Harada M, Tamada K, Tokuda N, Koikawa $Y$, Nakamura M, Nomoto K, Naito S: Antitumor activity of interleukin-I2 against murine bladder cancer. J Urol 2000, I 63:1549-1552

13. Chin JL, Kadhim SA, Batislam E, Karlik SJ, Garcia BM, Nickel JC, Morales A: Mycobacterium cell wall: an alternative to intravesical bacillus Calmette Guerin (BCG) therapy in orthotopic murine bladder cancer. J Urol 1996, I 56: I I89-I I93

14. Palli SR: Novel ecdysome receptor-based inducible gene expression systems. Molecular Therapy 200I, 3:

15. Leclerc GM, Boockfor FR, Faught W], Frawley LS: Development of a destabilized firefly luciferase enzyme for measurement of gene expression. Biotechniques 2000, 29:590-598

16. Wing KD: RH a nonsteroidal ecdysone agonist: effects on a Drosophila cell line. Science 5849, 241:467-469 
17. Coralli C, Cemazar M, Kanthou C, Tozer GM, Dachs GU: Limitations of the reporter green fluorescent protein under simulated tumor conditions. Cancer Res 200 I, 6 I:4784-4790

18. Bittner B, Thelly T, Isel H, Mountfield RJ: The impact of co-solvents and the composition of experimental formulations on the pump rate of the ALZET osmotic pump. Int J Pharm 2000, 205:195-198

19. Farquhar D, Cherif A, Bakina E, Nelson JA: Intensely potent doxorubicin analogues: structure-activity relationship. J Med Chem 1998, 41:965-972

Publish with BioMed Central and every scientist can read your work free of charge

"BioMedcentral will be the most significant development for disseminating the results of biomedical research in our lifetime." Paul Nurse, Director-General, Imperial Cancer Research Fund

Publish with BMC and your research papers will be:

- available free of charge to the entire biomedical community

- peer reviewed and published immediately upon acceptance

- cited in PubMed and archived on PubMed Central

- yours - you keep the copyright

Submit your manuscript here:

http://www.biomedcentral.com/manuscript/
BioMedcentral.com editorial@biomedcentral.com 